\title{
Solar cycle changes to planetary wave propagation and their influence on the middle atmosphere circulation
}

\author{
N. F. Arnold ${ }^{1,2}$, T. R. Robinson ${ }^{2}$ \\ ${ }^{1}$ Universities Space Research Association, Seabrook, M.D., USA \\ ${ }^{2}$ Department of Physics and Astronomy, University of Leicester, University Road, Leicester, LE1 7RH, UK
}

Received: 15 November 1996 / Revised: 2 July 1997 / Accepted: 4 July 1997

\begin{abstract}
Recent observations suggest that there may be a causal relationship between solar activity and the strength of the winter Northern Hemisphere circulation in the stratosphere. A three-dimensional model of the atmosphere between 10-140 km was developed to assess the influence of solar minimum and solar maximum conditions on the propagation of planetary waves and the subsequent changes to the circulation of the stratosphere. Ultraviolet heating in the middle atmosphere was kept constant in order to emphasise the importance of non-linear dynamical coupling. A realistic thermosphere was achieved by relaxing the upper layers to the MSIS-90 empirical temperature model. In the summer hemisphere, strong radiative damping prevents significant dynamical coupling from taking place. Within the dynamically controlled winter hemisphere, small perturbations are reinforced over long periods of time, resulting in systematic changes to the stratospheric circulation. The winter vortex was significantly weakened during solar maximum and western phase of the quasi-biennial oscillation, in accordance with reported $30 \mathrm{mb}$ geopotential height and total ozone measurements.
\end{abstract}

Key words. Meteorology and Atmospheric Dynamics (Climatology; Middle atmosphere dynamics; waves and tides)

\section{Introduction}

A number of empirical studies have been carried out which have indicated a correlation between sunspot activity and various aspects of the Earth's climate (Maunder, 1922; Eddy, 1976; Kelly, 1977; Kelly and

Correspondence to: N. F. Arnold at address 2
Wigley, 1990, 1992; Pittock, 1978; Sonett and Suess, 1984). However, exhaustive measurements of the emissions from the Sun show the total energy output has varied by only $0.1 \%$ over the past thirty years (Rottman, 1988). Changes are more pronounced at the shortwavelength extreme of the spectrum which accounts for a decreasing amount of the total. For example, variations in ozone due to ultraviolet flux changes over the complete 11-year solar cycle have been shown to be around 2-5\% (Gracia et al., 1984).

In contrast to the lower atmosphere, it is well known that the upper atmosphere varies considerably in response to changes in solar activity (e.g. Roble and Emery, 1983). Thermospheric temperatures may change by a factor of two during the solar cycle. The temperature differences between solar minimum and maximum (solar flux at $2800 \mathrm{MHz}, 10.7 \mathrm{~cm}$, i.e. F10.7 = 70 and $220 \mathrm{Wm}^{-2} \mathrm{~Hz}^{-1}$ respectively) between altitudes of 60 and $120 \mathrm{~km}$, derived from the empirical model based on mass spectrometer and incoherent scatter data (MSIS 90E, Hedin, 1983, 1991) are given in Fig. 1.

Observations indicate that the stratosphere is also able to respond to changes in the solar flux. Kodera and Yamazaki (1990) and Hood et al. (1993) have reported significant zonal wind variations in the Northern Hemisphere winter through the solar cycle. Labitzke and van Loon $(1988,1994,1995)$ used over 30 years of daily observations to obtain a correlation between solar variability and the stratospheric $30 \mathrm{mb}$ geopotential height field.

Labitzke and van Loon (1993) proposed that direct heating is the key mechanism, but noted that the calculated radiative forcing is far too small to explain the observations. They also demonstrated that the quasi-biennial oscillation (QBO) plays an important role in determining the response of the middle atmosphere to climatic variations. The correlation was improved by distinguishing between the eastward and westward phase components. Observations of total ozone over Iceland have shown similar tendencies (Bjarnason et al., 1993). 


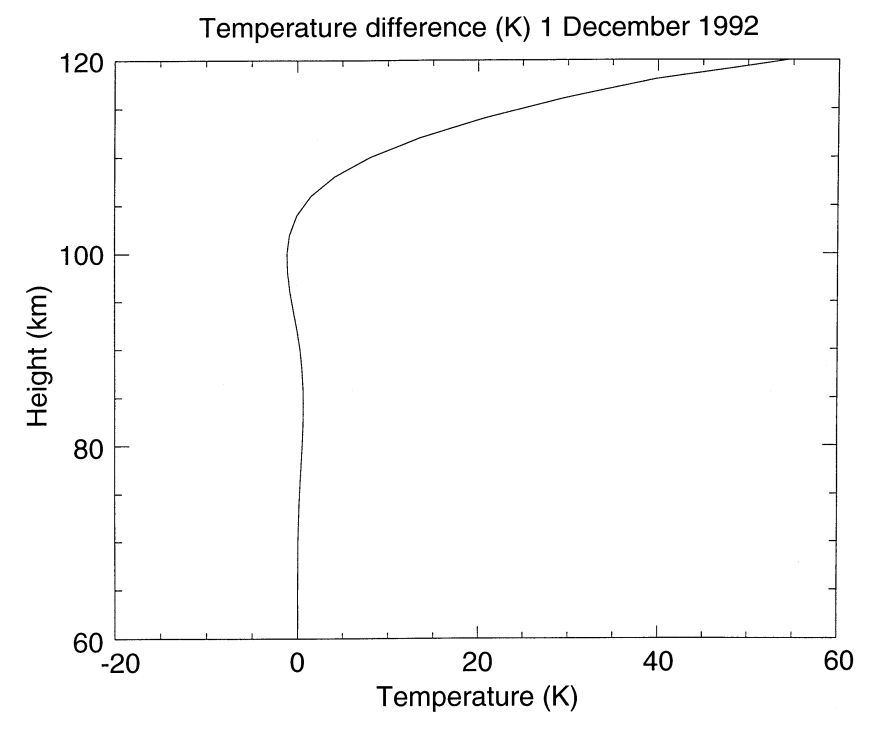

Fig. 1. An altitude profile of globally averaged temperature difference (K) between solar minimum $\left(\mathrm{F} 10.7=70 \mathrm{Wm}^{-2} \mathrm{~Hz}^{-1}\right)$ and solar maximum $\left(\mathrm{F} 10.7=220 \mathrm{Wm}^{-2} \mathrm{~Hz}^{-1}\right)$ assuming quiet geomagnetic conditions using the MSIS 90 model atmosphere

Three-dimensional simulations have been performed where the solar heating has been allowed to vary. Haigh (1994, 1996) demonstrated that consistent and statistically significant changes to the Earth's middle atmosphere could be produced, but with amplitudes that were considerably smaller than those that have been reported. Kodera et al. (1990) and Balachandran and Rind (1995) were able to achieve the desired amplitude response, but only by assuming unrealistically large ozone and ultraviolet heating variations respectively.

Detailed two-dimensional modelling studies have predicted changes in temperature and ozone of less than $3 \%$ throughout most of the atmosphere below $70 \mathrm{~km}$ (Huang and Brasseur, 1993). Brasseur (1993) suggested that an additional coupling mechanism between the radiation, dynamics and chemistry was required.

An important consideration in any link between the upper and lower atmospheres is one of energetics. The thermosphere accounts for less than one part in a million of the mass of the atmosphere. The amplitude of any disturbance propagating downwards in the atmosphere is reduced by a factor of ten over a couple of scale heights due to the exponential rise in pressure towards the surface of the Earth. Simple linear perturbation theory would suggest that any mechanism which transferred sufficient energy from the thermosphere to the lower atmosphere would be highly improbable. Therefore, it is necessary to consider non-linear dynamical systems where outcomes are extremely sensitive to variations in initial and boundary conditions (Lorenz, 1963, 1969).

Holton (1994) has suggested that the Northern Hemisphere may have two stable atmospheric states, one with strong eddy motions and warm polar temperatures and the other with weaker eddies and colder temperatures. The equatorial $\mathrm{QBO}$ changes the probability that the atmosphere may be in one or other of the two regimes. Weak changes in the solar cycle forcing, or indeed other ten-to-twelve year processes, may be sufficient to alter the likelihood of being in one state or the other. At the end of his paper he indicated that he favoured changes to the 'stratospheric wave guide' which would in turn influence the climate system.

In this study we shall examine the extent to which the lower thermosphere affects the propagation of planetary waves. Earlier work has shown that changes in the mesosphere due to gravity wave drag exerts a strong influence on the stratospheric circulation through 'downward control' (Haynes et al., 1991), so it is reasonable to assume that smaller effects should exist due to higher altitude phenomena. We have deliberately ignored the contribution of direct solar heating variability in the middle atmosphere, mentioned already, to simplify the interpretation of the results.

A well-established mechanistic model of the middle atmosphere (Austin and Butchart, 1992; O'Neil and Pope, 1988) has been extended into the lower thermosphere and is described in Sect. 2. The results of solar minimum and solar maximum experiments are presented in Sect. 3. The importance of planetary waves is explored in Sect. 4 along with a brief discussion of the possible contribution from gravity wave coupling. A summary and future developments are provided in Sect. 5.

\section{The extended UK Meteorological Office stratosphere mesosphere model (ESM)}

The original UK Meteorological Office stratosphere mesosphere model (SMM) was developed to provide good simulations of the middle atmosphere without the complexities of the tropospheric hydrological cycle (e.g. O'Neil and Pope, 1988). It is an explicit 'leap-frog' time stepping grid-point model such that both energy and momentum are conserved. The lower boundary at $314 \mathrm{mb}$ was prescribed by geopotential height fields derived from the Stratospheric Sounding Unit (see Miller et al., 1980 for a review of the instrument and data), thereby allowing realistic planetary wave forcing. With the addition of transport and chemistry, it has proved to be a useful tool for ozone research (Austin and Butchart, 1992; Austin et al., 1992).

For this study, the model resolution was set at $5^{\circ}$ by $5^{\circ}$ in the horizontal $(555 \mathrm{~km}$ on a great circle) and 0.2878 scale heights in the vertical. In the middle atmosphere the vertical resolution is around $2 \mathrm{~km}$, but is progressively reduced in the lower thermosphere. Therefore the top of the model could be raised from $70 \mathrm{~km}$ to $140 \mathrm{~km}$ without being prohibitively expensive computationally. A time step of $90 \mathrm{~s}$ was chosen to ensure numerical stability, especially near the poles.

The radiation scheme developed by Shine (1987) provides a detailed treatment of ultraviolet and infrared heating and cooling in the middle atmosphere up to $70 \mathrm{~km}$. Above $90 \mathrm{~km}$ the ultraviolet heating rates of Strobel (1978) were extended up to $140 \mathrm{~km}$. Following Garcia and Solomon (1983), a constant diabatic cooling term was derived by globally averaging over a constant 
log-pressure surface. A Newtonian cooling scheme was then applied to further constrain the model temperature.

The reference field for the Newtonian relaxation was the zonal mean of the empirical MSIS temperature model (Hedin, 1983, 1991). MSIS values are derived from a large number of temperature measurements over a number of years and can be specified as a function of solar activity. It can only be statistically representative of certain seasons and solar flux conditions rather than any specific date. In its favour, it is not necessary to simulate the detailed heating and cooling processes which are important in the lower thermosphere, thus making the problem tractable.

Quiet geomagnetic conditions $(\mathrm{Ap}=1.0)$ were chosen throughout the simulations. Gravity wave drag was simulated using Rayleigh friction. An additional component to account for ion drag in the thermosphere was also included. Molecular diffusion in the lower thermosphere was treated by using a Laplacian smoothing operator at these levels. Eddy diffusion was represented by a Shapiro (1970) filter.

\section{Simulations of solar minimum and solar maximum conditions}

The MSIS-90E empirical model provided the initial temperature fields for the simulations of solar minimum and solar maximum conditions. The horizontal winds were taken from the horizontal wind model (Hedin et al., 1993). Lower boundary data were available for the period 1st December 1992 till 28th February 1993. This coincided with a western phase of the QBO at the equator above $18 \mathrm{~km}$. The model runs required at least 10 days to achieve thermal wind balance and to dampen away the strong shears that were generated at the beginning of the integration. During this period, the lower boundary forcing was allowed to evolve from the zonal mean to its true distribution to minimise numerical instabilities.

Figure $2 \mathrm{a}$ shows the zonally averaged temperature from the MSIS model for February 19th 1993. These results can be compared with those obtained from the ESM simulation in Fig. 2b, 80 days into the integration. The former is a multi-year average of observations, whilst the latter is driven by planetary wave activity from the winter of $1992 / 93$. This period was chosen for its pronounced planetary wave activity. Therefore, one would not expect the details to be exactly the same. Figure 3 shows the zonal mean zonal winds from the same model day. As the plot was taken from a single time step, some inertial instability was present near the equator, but this would disappear in a longer term average of the data.

The model appears to do a reasonable job in the middle atmosphere of capturing the bulk of its features. The upper air jets are closed around $90 \mathrm{~km}$ by the gravity wave momentum deposition and there is no strong discontinuity in the fields around $70-90 \mathrm{~km}$ where the two heating regimes are merged. Both simulations were integrated successfully for over three
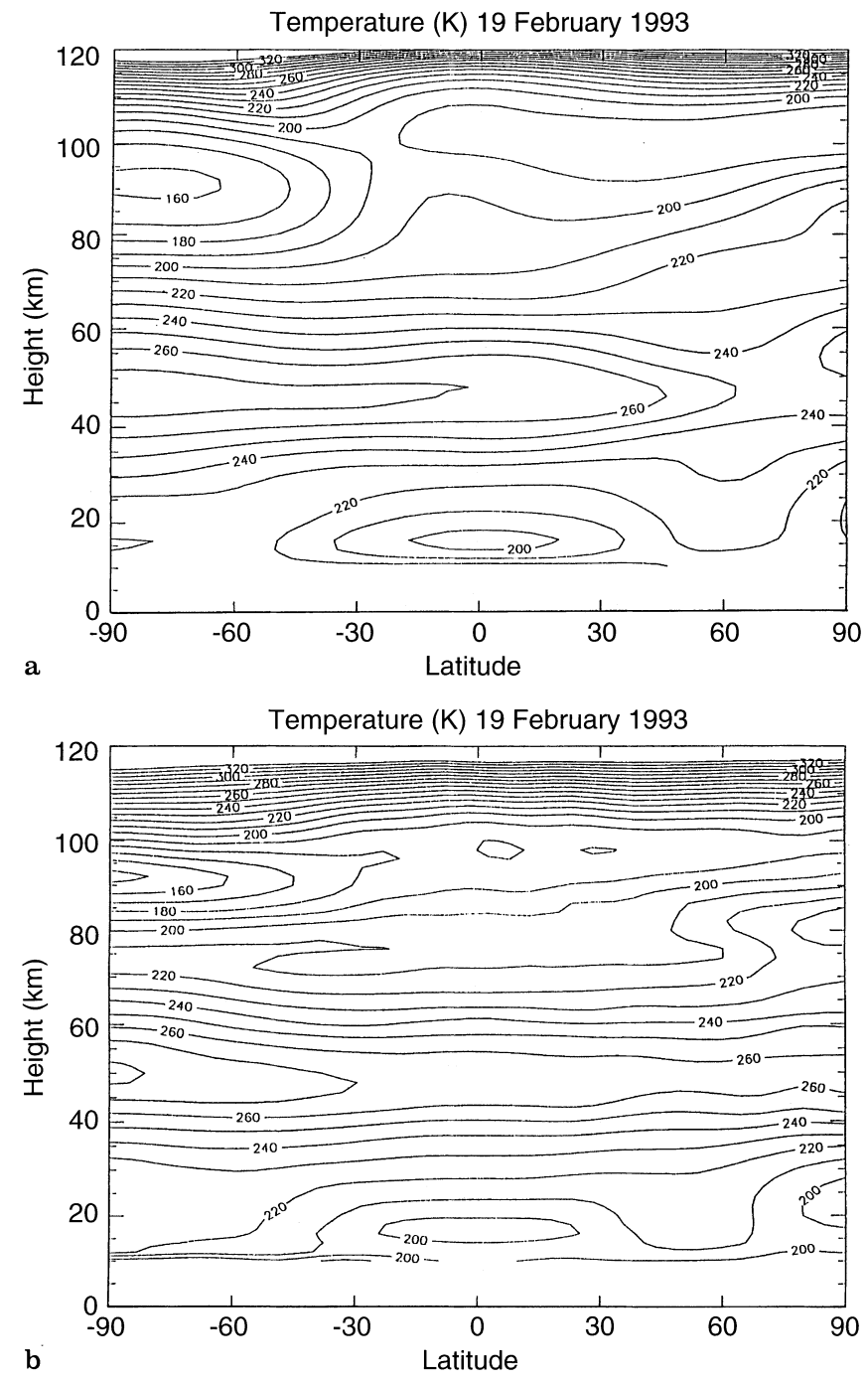

Fig. 2a,b. Zonally averaged temperature at solar minimum for 19th February, 1993 a from the MSIS-90E model atmosphere, b from the extended Meteorological Office stratosphere mesosphere model for the same date integrated from December 1st 1992

months without diverging significantly from the typical results shown here.

A simple means of comparing solar minimum and solar maximum conditions is to take the zonal mean of the differences between the two simulations for the same period of integration. Figure $4 \mathrm{a}$ shows the temperature differences after sixty days. In the summer hemisphere, there is very little noticeable variation in the temperature between the two cases, even considering numerically generated noise. The Northern Hemisphere has been altered globally above $30 \mathrm{~km}$ by several degrees. The most pronounced effect can be seen in the zonal wind data (Fig. 4b). Differences of the order of $20 \mathrm{~ms}^{-1}$ have been generated around the edge of the winter vortex. This can be partially accounted for by the fact that the steep gradient in the winds will result in a small vortex displacement producing an amplified response.

A more detailed view of the localised nature of the perturbations can be found in Fig. 5a, b. Cross sections of the temperature and zonal wind were taken for the 


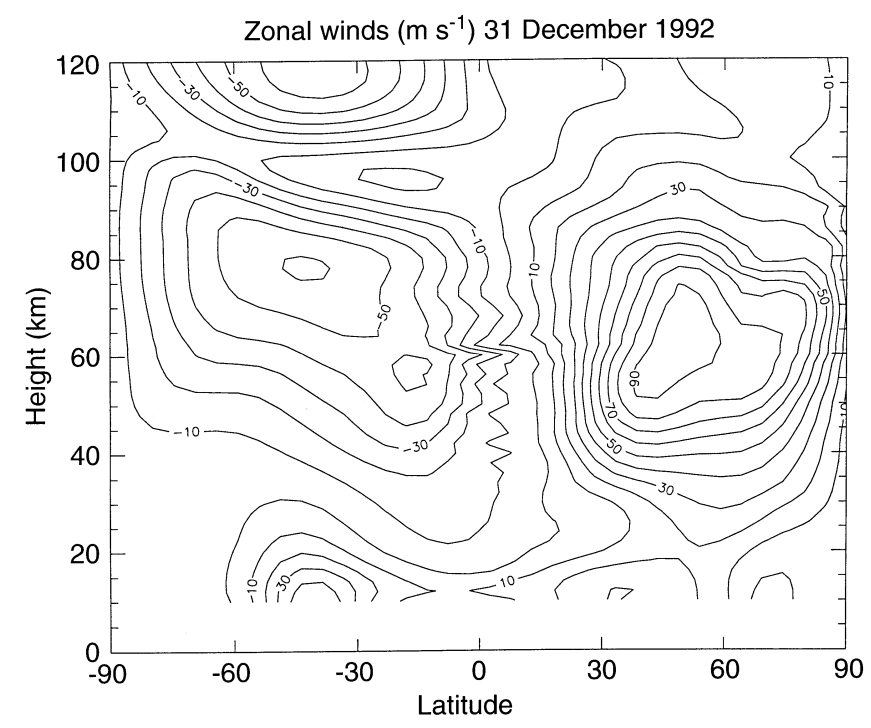

Fig. 3. Zonally averaged zonal winds at solar maximum for 31 st December, 1992 from the EMM

same model day at an altitude of around $30 \mathrm{~km}$. Over the Ural mountains, the temperature difference between solar minimum and solar maximum was around $9 \mathrm{~K}$, three times higher than the zonal mean value. Stronger departures could be found in the zonal wind fields in the Northern Hemisphere. However, the activity tailed off rapidly into the Southern Hemisphere.

These experiments were repeated with the lower boundary forcing turned off. Figure 6 suggests that the differences discussed could not be accounted for by numerical effects alone. In addition, the difference between the Southern and Northern Hemispheres may be attributed to the strength of the planetary wave forcing.

Any experiment which relies upon non-linear processes, produces results which can be either choatic or deterministic. To assess the relative importance of these two factors in the experiment, we modified the starting dates and the level of the solar forcing by small increments. It was found that changes of a few percent in the forcing produced small temperature and wind field differences, which were qualitatively rather variable in the Northern Hemisphere whilst very little was seen in the Southern Hemisphere (not shown). More consistent circulation differences were obtained when the perturbation was of the order of $10 \%$.

Figure 7 illustrates the difference in zonally averaged zonal mean winds after 60 days when the solar fluxes were 210 and $180 \mathrm{Wm}^{-2} \mathrm{~Hz}^{-1}$ respectively. Note that there is good qualitative agreement with the solar maximum-solar minimum case, but also that the magnitude is a factor of ten lower than before. It appears that the results are robust above a noise threshold which is considerably lower than that required to obtain a clear solar cycle signal. One further implication is that in this model at least, day to day fluctuations in the solar flux may be poorly correlated with the atmospheric circulation, but there is still the possibility that a coherent response to the 27-day cycle may be detectable.

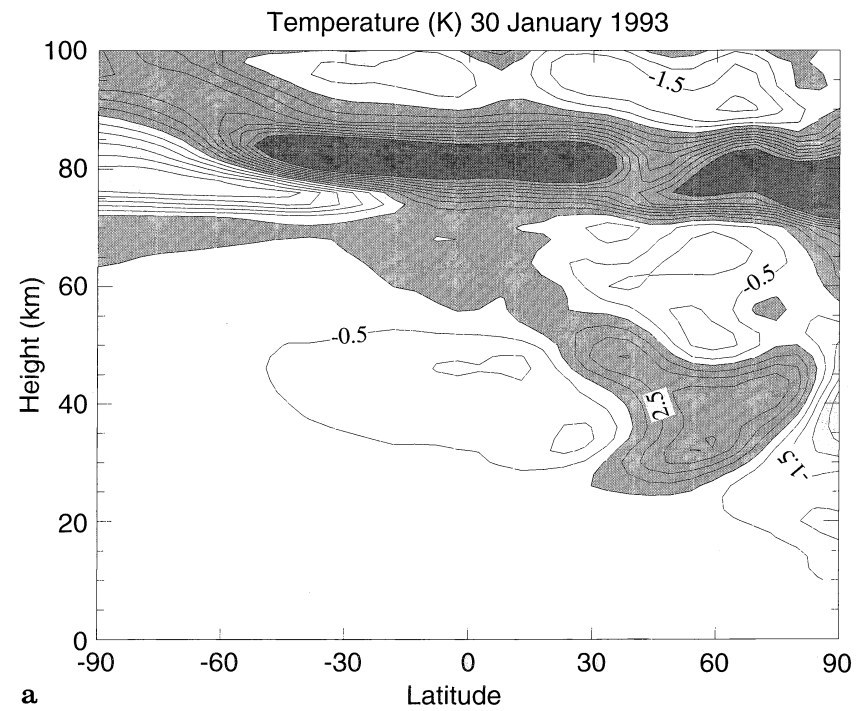

a

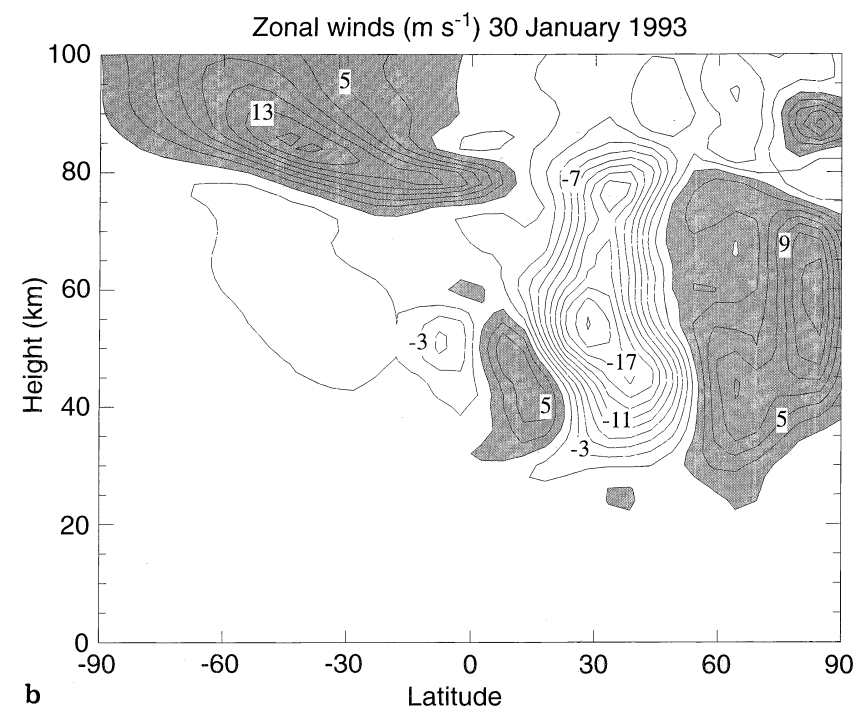

Fig. 4a,b. Zonally averaged differences in a temperature $(\mathrm{K})$, b zonal velocity $\left(\mathrm{ms}^{-1}\right)$ from the EMM after 60 days of model integration between solar maximum and solar minimum. Light shading denotes values of $0.5 \mathrm{~K}$ and $1 \mathrm{~ms}^{-1}$ and more respectively and dark shading greater than $9.5 \mathrm{~K}$

\section{Planetary and gravity wave coupling processes}

To gain further insight into the behaviour of the planetary waves, Northern Hemisphere Eliassen-Palm (EP) fluxes were computed for January 30th (see for example Andrews et al., 1986, for a comprehensive explanation of this quantity). The fluxes were divided by the air density to allow the 'wave activity' vectors to be seen more readily. Where the amplitudes and divergences of the vectors are large, significant energy and momentum is deposited into the mean flow at that point. At solar minimum (Fig. 8a), there were relatively weak EP-fluxes at mid-latitudes with generally vertically upward flow north of $60^{\circ} \mathrm{N}$ and equator ward flow south of $40^{\circ} \mathrm{N}$. This corresponds to relatively weak interactions between the planetary waves and the mean circulation. At solar maximum (Fig. 8b), the middle atmosphere fluxes were considerably stronger with 

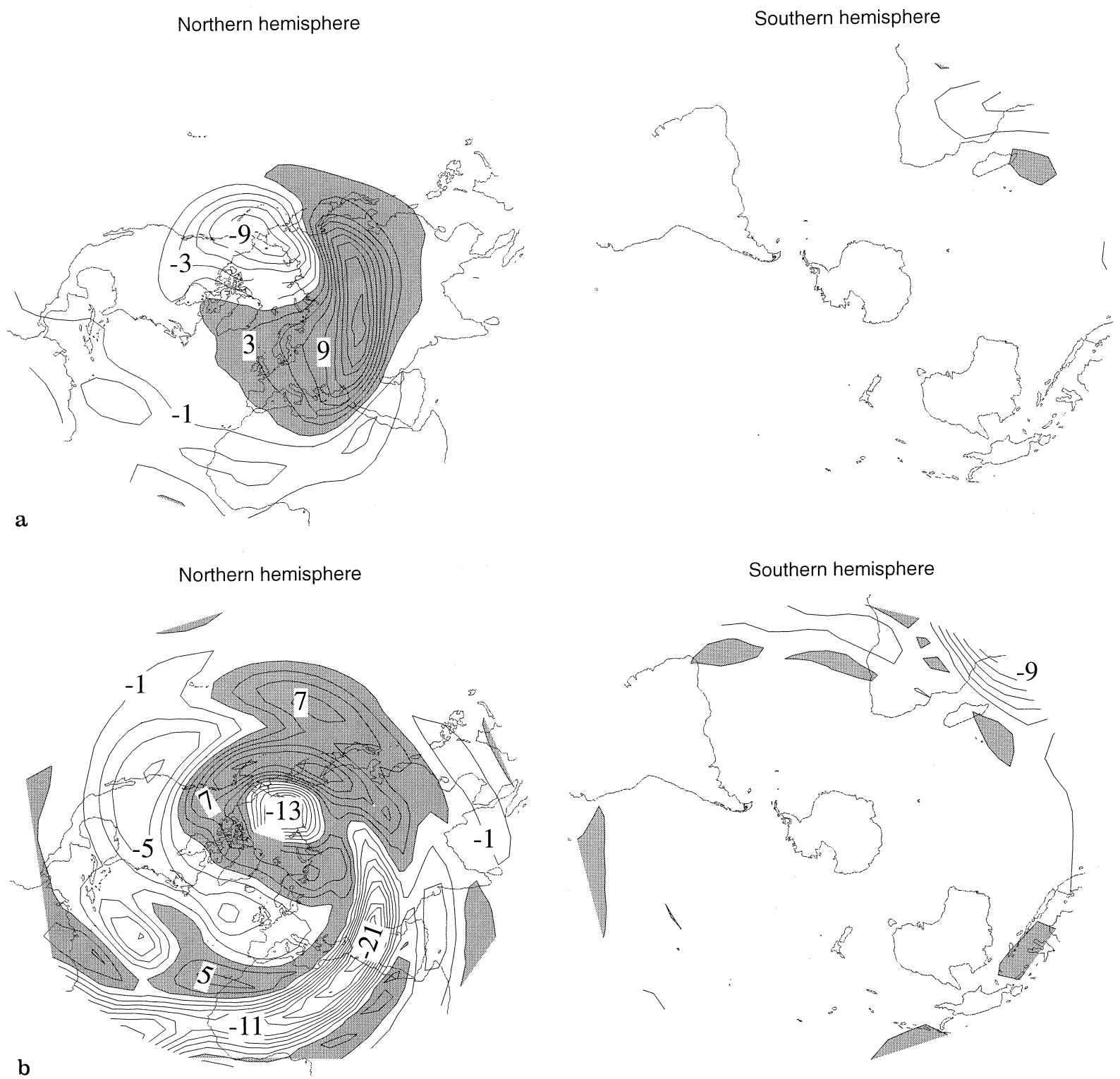

Fig. 5. As Fig. 4 but showing the polar stereographic cross sections at $\sim 30 \mathrm{~km}$

almost vertical flow at mid-latitudes below $45 \mathrm{~km}$ and equatorial flows above $50 \mathrm{~km}$ south of $40^{\circ} \mathrm{N}$. Under these conditions, the planetary waves are expected to exert a strong influence on the circulation around the polar vortex. As the original amplitudes of the EP fluxes were very small in the Southern Hemisphere, the differences were similarly unremarkable (not shown).

The rate of divergence between the difference fields at solar minimum and solar maximum did not occur evenly. Changes occurred more readily during a small number of relatively large forcing events, such as minor and major stratospheric warmings, rather than a large number of small perturbations. The former were more effective at propagating deep into the mesosphere. With relatively long radiative time scales, the waves were not damped before significant irreversible interactions had taken place with the circulation.

Once changes in the thermosphere had started to impinge upon the stratosphere, further changes occurred quite readily. All subsequent planetary waves were deflected from the paths that they would have taken in the absence of increased solar activity, bringing about still further departures from this state. The cycle is completed when further planetary waves, entering the upper mesosphere, will themselves have different properties. The coupling thus creates a feedback which may be either positive or negative, depending upon the details of the waves and the circulation patterns.

The saturation of gravity waves in the upper mesosphere provides an important source of vertical diffusion and should enhance the results obtained by planetary wave coupling alone. Mengel et al. (1995) used a numerical model based on the implementation of Hines' physical parametrisation where the largest fluxes were obtained near the mesopause (approximately $90 \mathrm{~km}$ ), close to the region where solar flux changes are important. Any perturbations to the temperatures would affect the zonal winds, which would influence 

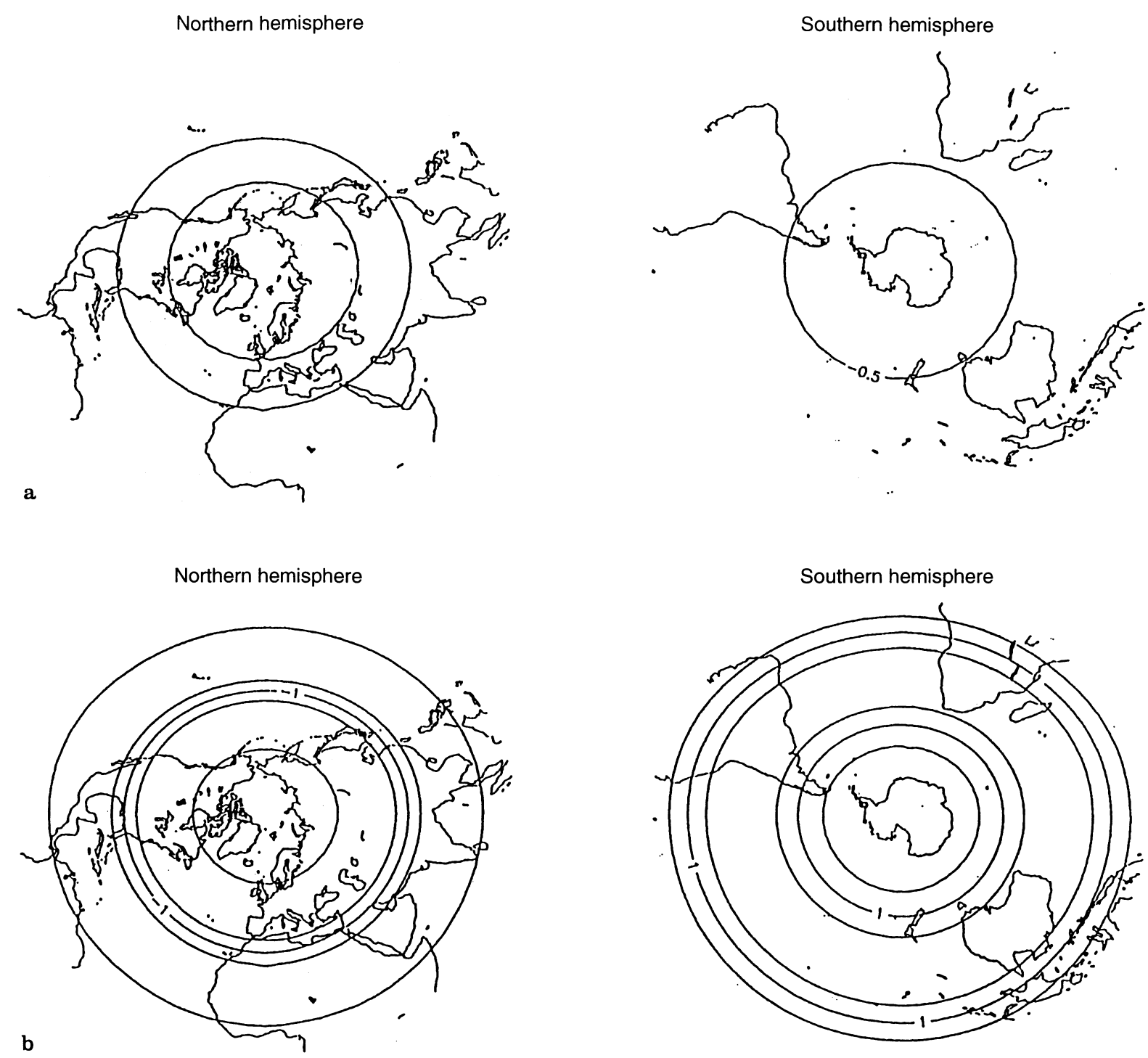

Fig. 6. As Fig. 5 but with no planetary wave forcing at the lower boundary

the gravity wave deposition. Through the process of 'downward control' (Haynes et al., 1991) the mesospheric gravity waves would, in turn, influence the stratospheric circulation.

Detailed studies of the interactions between mesospheric gravity waves, planetary waves and the mean circulation have yet to be performed, so it is beyond the scope of this work to examine the role of these waves in the coupling process. However, recent work by Bristow et al. (1996) suggests that gravity waves may be important. Using observations from the Goose Bay $\mathrm{HF}$ radar, they were able to demonstrate that the relative frequency of the transmission of Earth-reflected gravity waves back into the thermosphere was affected by the thermal gradient at the mesopause, which varied according to the seasonal cycle. It is not unreasonable to speculate that as the thermal gradient changes through the solar cycle, similar effects will be seen in the momentum rates. This issue will be addressed once the Hines (1991) gravity wave scheme has been implemented for this purpose.

\section{Conclusion}

Understanding natural climate change is an important factor in assessing anthropogenic modifications (IPCC, 1990, 1996). The processes by which changes in solar activity affect the Earth's lower atmosphere are, by the nature of the system, rather complex. Simple direct means of reproducing the observations have failed so far, leading to speculation that indirect coupling processes are also required. Waves clearly play an important role in this and one small aspect of this has been addressed here.

In this study we have presented a relatively simple, mechanistic, three-dimensional model of the atmosphere between 10 and $140 \mathrm{~km}$ which is able to respond in a controlled fashion to changes induced by the 11 y solar cycle. The level of planetary wave activity is shown to be a key process in determining the extent to which the stratosphere is able to respond to changes in the lower thermosphere. Longer radiative relaxation times in the winter hemisphere, compared with the summer, allow 


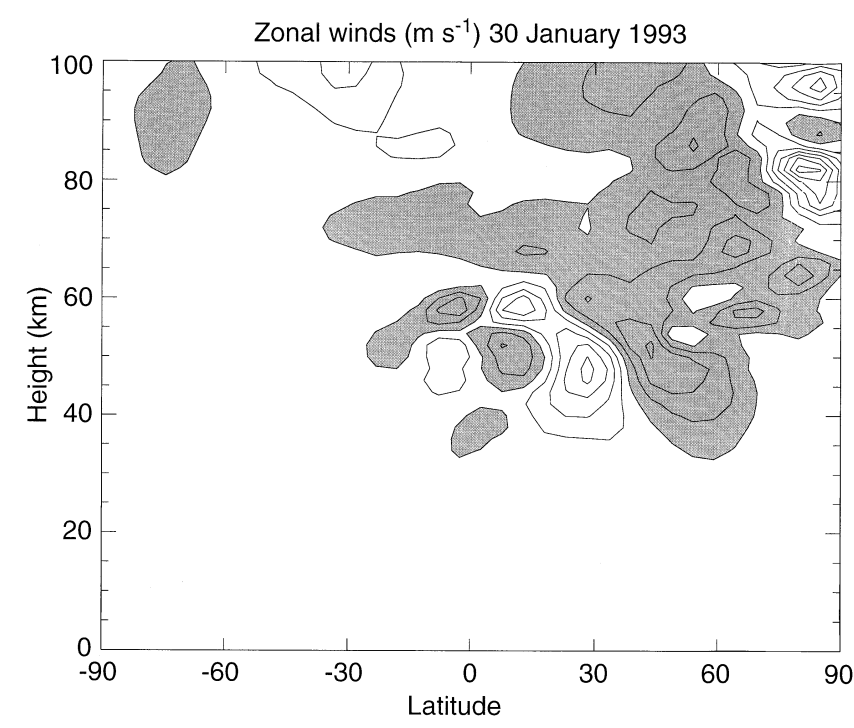

Fig. 7. Zonally averaged differences in zonal mean winds $\left(\mathrm{ms}^{-1}\right)$ from the ESM after 60 days of model integration between solar flux levels of $\mathrm{f} 10.7=210$ and $180 \mathrm{wm}^{-2} \mathrm{H}_{2}^{-1}$. Contour intervals are $2 \mathrm{~ms}^{-1}$ and shading denotes values greater than $1 \mathrm{~ms}^{-1}$

the effects of altered planetary wave propagation paths to accumulate. In addition, the changes to the middle atmosphere begin to affect the propagation of planetary waves themselves and this becomes an important mechanism in amplifying smaller differences.

The lower boundary planetary wave forcing was taken during the western phase of the quasi-biennial oscillation. At the time, there was a moderate level of solar activity (between 100 and $170 \mathrm{Wm}^{-2} \mathrm{~Hz}^{-1}$ with a three month mean of around $130 \mathrm{Wm}^{-2} \mathrm{~Hz}^{-1}$ ). There was a series of warmings, including a major warming event. Artificially applying high solar activity was shown to promote strong planetary wave activity, whilst low solar activity reduced it considerably.

Much further work is required to refine the model, especially with respect to gravity waves. Improvements to the upper mesospheric physics, such as the replacing the relatively crude Newtonian heating and MSIS approach, would improve the simulation above $75 \mathrm{~km}$, albeit at the expense of adding to the complexity of the model and its interpretation. Simulations are being performed to attempt to detect a stratospheric signal from the 27-day solar cycle and periods of intense geomagnetic activity. A logical progression will be to run the model during an eastern phase of the $\mathrm{QBO}$ when there was no Northern Hemisphere major warming.

Acknowledgements. The authors wish to thank J. Austin and N. Butchart for making the stratosphere mesosphere model available to us and A. Hedin for providing the MSIS 90E and HWM models. The continued support of T. Jones, M. Schoeberl, M. Kalb and R. Hartle made this work possible. Many important contributions to the upper atmospheric science were made by H. Mayr and D. Pesnell. N. Arnold was supported by NASA grant NAS 5-32484. We are grateful to two anonymous referees for their very constructive comments on the original manuscript.

Topical Editor F. Vial thanks L. L. Hood and R. Harwood for their help in evaluating this paper.
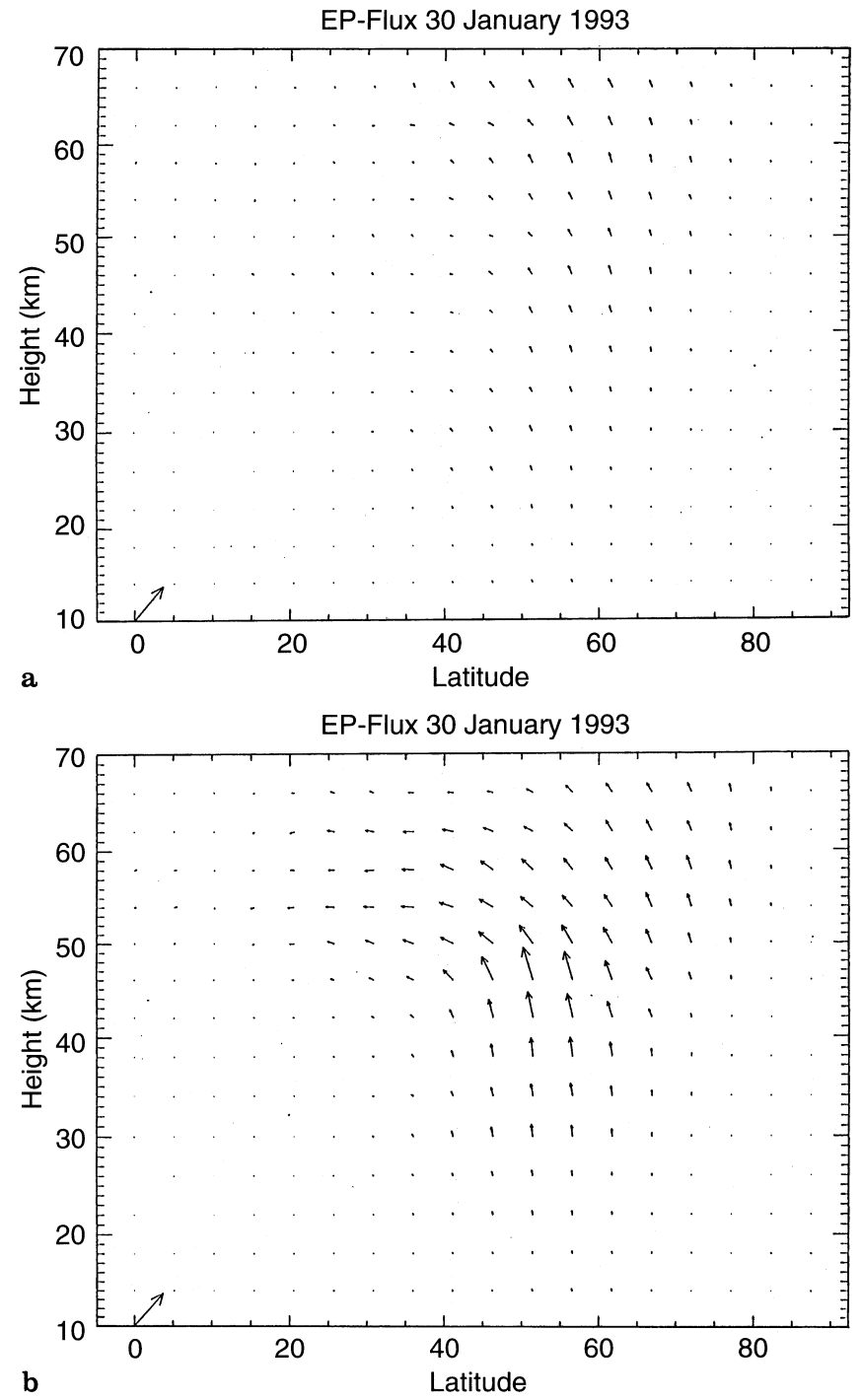

Fig. 8a,b. Northern Hemisphere normalised Eliassen-Palm fluxes scaled by $1 / \rho$ for a solar minimum and $\mathbf{b}$ solar maximum conditions

\section{References}

Andrews, D. G., J. R. Holton, and C. B. Leovy, Middle atmosphere dynamics, Academic Press, Orlando pp 489, 1987.

Austin, J., and N. Butchart, A three-dimensional modeling study of the influence of planetary wave dynamics on polar ozone photochemistry, J. Geophys. Res., 97, 10 165-10 186, 1992.

Austin, J., N. Butchart, and K. P. Shine, Possibility of an Arctic ozone hole in a doubled- $\mathrm{CO}_{2}$ climate, Nature, 360, 221-225, 1992.

Balachandran, N. K., and D. Rind, Modeling the effects of UV variability and the QBO on the troposphere-stratosphere system. Part I: the middle atmosphere. J. Climate, 8, 2058-2079.

Bjarnason, G. G., Ö. E. Rögnavaldsson, T. I. Sigfússon, T. Jakobsson, and B. Thorkelsson, Total ozone variations at Reyjavík since 1957, J. Geophys. Res., 98, 23 059-23 077, 1993.

Brasseur, G. P., The response of the middle atmosphere to long term and short term solar variability: a two-dimensional model, J. Geophys. Res., 98, 23 079-23 091, 1993.

Bristow, W. A., R. A. Greenwald, and J. P. Villain, On the seasonal dependence of medium-scale atmospheric gravity waves in the upper atmosphere at high latitudes, J. Geophys. Res., 101, 15 685-15 700, 1996. 
Eddy, J. A., The Maunder minimum, Science, 192, 1189-1202, 1976.

Garcia, R. R., and S. Solomon, A numerical model of the zonally averaged dynamical and chemical structure of the middle atmosphere, J. Geophys. Res., 88, 1379-1400, 1983.

Garcia, R. R., S. Solomon, R. G. Roble, and D. W. Rusch, A numerical response of the middle atmosphere to the 11-year solar cycle, Planet. Space Sci., 32, 411-423, 1984.

Haigh, J. D., The role of stratospheric ozone in modulating the solar radiative forcing of climate, Nature, 370, 544-546, 1994.

Haigh, J. D., Science, 272, 981-984, 1996.

Haynes, P. H., C. J. Marks, M. E. McIntyre, T. G. Shepherd, and K. P. Shine, On the downward control of extratropical diabatic circulations by eddy-induced mean zonal forces, J. Atmos. Sci., 48, 651-679, 1991.

Hedin, A. E., A revised thermospheric model based on mass spectrometer and incoherent scatter data: MSIS-83, J. Geophys. Res., 88, 10 170-10 188, 1983.

Hedin, A. E., Neutral atmosphere empirical model from the surface to the lower exosphere: MSISE, J. Geophys. Res., 96, 11591172, 1991.

Hedin, A. E., E. L. Fleming, A. H. Manson, F. J. Schmidlin, S. K. Avery, and S.J. Franke, Empirical wind model for the middle and lower atmosphere. Part 1: local time average, NASA Techn. Memo. 104581, 1993.

Hines, C. O., The saturation of gravity waves in the middle atmosphere. Part II: development of Doppler Spread theory, $J$. Atmos. Sci., 48, 1360-1369, 1991.

Holton, J. R., The quasi-biennial oscillation in the Earth's atmosphere and its links to longer period variability, in NATO ASI Series 1: Global Environmental Change, 25, The solar engine and its influence on terrestrial atmosphere and climate, Ed. E. Nesme-Ribes, Springer-Verlag, Berlin Heidelberg New York, pp 259-273, 1994.

Hood, L. L., J. L. Jirikowic, and J. P. McCormack, Quasi-decadal variability of the stratosphere - influence of long-term solar ultraviolet variations, J. Atmos. Sci., 50, 3941-3958, 1993.

Huang, T. Y. W., and G. P. Brasseur, Effect of long-term solar variability in a two-dimensional interactive model of the middle atmosphere, J. Geophys. Res., 98, 20 413-20 427, 1993.

Intergovernmental Panel on Climate Change, Climate change: the IPCC scientific assessment, Ed. J. T. Houghton, G. J. Jenkins and J. J. Ephraums, University Press, Cambridge, pp 365, 1990.

Intergovernmental Panel on Climate Change, Climate change 1995: the science of climate change Ed. J. T. Houghton, L. G. Meira Filho, B. A. Callander, N. Harris, A. Kattenberg and K. Maskell, University Press, Cambridge, pp 572, 1996.

Kelly, P. M., Solar influence on north Atlantic mean sea level pressure, Nature, 269, 320-322, 1977.

Kelly, P. M., and T. M. L. Wigley, The influence of solar forcing trends on global mean temperature since 1861, Nature, 347, 460-462, 1990.

Kelly, P. M., and T. M. L. Wigley, Solar cycle length, greenhouse forcing and global climate, Nature, 360, 328-330, 1992.
Kodera, K., and K. Yamazaki, Long-term variations of upper stratospheric circulation in the Northern Hemisphere in December, J. Meteorol. Soc. Japan, 68, 101-105, 1990.

Kodera, K., M. Chiba, and K. Shibata, A general circulation model study of the solar and QBO modulation of the stratospheric circulation during the Northern Hemisphere winter, Geophys. Res. Lett., 18, 1209-1212, 1991.

Labitzke, K., and H. van Loon, Association between the 11-year solar cycle, the QBO and the atmosphere. Part 1: the troposphere and stratosphere in the Northern Hemisphere in winter, J. Atmos. Terr. Phys., 50, 197-206, 1988.

Labitzke, K., and H. van Loon, Some recent studies of probable connections between solar and atmospheric variability, Ann. Geophysicae, 11, 1084-1094, 1993.

Labitzke, K., and H. von Loon, A probable connection between solar and atmospheric decadal variability, in The sun as a variable star. (Eds. J. M. Pap, C. Frohlich, H. S. Hudson and S.K. Solanki. Cambridge University Press, pp 330-338, 1994.

Labitzke, K., and H. van Loon, Connection between the troposphere and stratosphere on a decadal scale, Tellus, 47A, 275286, 1995.

Lorenz, E. N., Deterministic nonperiodic flow, J. Atmos. Sci., 20, 130-141, 1963.

Lorenz, E. N., The predictability of a flow which possesses many scales of motion, Tellus, 21, 289-307, 1969.

Maunder, E. W., The prolonged sunspot minimum 1645-1715, J. Br. Astron. Assoc., 32, 140-145, 1922.

Mengel, J. G., H. G. Mayr, K. L. Chan, C. O. Hines, C. A. Reddy, N. F. Arnold, and H. S. Porter, Equatorial oscillations in the middle atmosphere generated by small-scale gravity waves, Geophys. Res. Lett., 22, 3029-3030, 1995.

Miller, D. E., J. L. Brownscombe, G. P. Carruthers, D. R. Pick, and K. H. Stewart, Operational temperature sounding of the stratosphere, Philos. Trans. R. Soc. London, A292, 65-71, 1980.

O'Neill, A., and V. D. Pope, Simulations of linear and non-linear disturbances in the stratosphere. Q. J. R. Meteoral. Soc., 114, 1063-1110, 1988.

Pittock, A. B., A critical look at long-term sun-weather relationships, Rev. Geophys. Space Phys., 16, 400-420, 1978.

Roble, R. G., and B. A. Emery, On the global mean temperature of the thermosphere, Planet. Space Sci., 31, 594-614, 1983.

Rottman, G. J., Observations of solar UV and EUV variability, Adv. Space Res., 8, 53-66, 1988.

Shapiro, R., Smoothing, filtering and boundary effects, Rev. Geophys. Space Phys., 8, 359-387, 1970.

Shine, K. P., The middle atmosphere in the absence of dynamical heat fluxes, Q. J. R. Meteorol. Soc., 113, 603-633, 1987.

Sonett, C. P., and H. E. Suess, Correlation of bristlecone pine ring widths with atmospheric ${ }^{14} \mathrm{C}$ variations: a climatic-sun relation, Nature, 307, 141-143, 1984.

Strobel, D. F., Parametrization of the atmospheric heating rate from 15 to $120 \mathrm{~km}$ due to $\mathrm{O}_{2}$ and $\mathrm{O}_{3}$ absorption of solar radiation, J. Geophys. Res., 83, 6225-6230, 1978. 